\title{
A Stochastic Model for Highway Accident Predictions with Winter Data
}

\author{
Chen Lin, Michael Jong Kim and Viliam Makis ${ }^{*}$
}

\author{
Department of Mechanical and Industrial Engineering, University of Toronto, 5 King's College Road, Toronto, ON, \\ M5S3G8, Canada
}

\begin{abstract}
In this paper, we consider the problem of modeling and predicting highway accidents in the presence of randomly changing winter driving conditions. Unlike most accident prediction models in the literature, which are typically formulated in a static (e.g. regression models) or discrete time (e.g. time-series models) setting, we propose a continuoustime stochastic model to describe the relation between highway accidents and winter weather dynamics. We believe this to be a more natural way to describe discrete-event highway accidents that occur in continuous-time. In particular, the accident counting process is viewed as a non-homogeneous Poisson process (NHPP) with an intensity function that depends on a (Markovian) weather process. Such a model is known in the stochastic process literature as a Markovmodulated Poisson process (MMPP) and has been successfully applied to queuing and telecommunications problems. One main advantage of such an approach, is its ability to provide explicit closed-form prediction formulae for both weather and accidents over any future time horizon (i.e. short or long-term predictions). To illustrate the effectiveness of the proposed stochastic model, we study a large winter data set provided by Ministry of Transportation of Ontario (MTO) that includes motor vehicle accidents on Highway 401, the busiest highway in North America.
\end{abstract}

Keywords: Highway accidents, Winter driving conditions, Stochastic modeling, Markov-modulated Poisson process, Maximum likelihood estimation, Prediction, Operational performance functions

\section{INTRODUCTION}

Generalized linear regression models and generalized linear mixed regression models have been widely considered to model crash data and analyze the effects of different factors (e.g. weather) on safety. Typical models include the binomial, Poisson, Poisson-Gamma, zero-inflated Poisson (ZIP), beta-binomial, multinomial and mixture models [1-8]. For a recent comprehensive review of this literature see [9]. In such models, explanatory variables are considered as deterministic variables that affect the frequency of accidents. However, when explanatory variables are autocorrelated and/or stochastically change over time, predicting future outcomes based on observable present conditions is not always suitable in the standard regression analysis framework.

To account for this time dependency, some researchers have considered regression models applied to time-series data [10-14]. For example, [15] proposed an ARIMA model to forecast the highway collision frequencies. [10] proposed a so-called inter-valued autoregressive (INAR) Poisson model to improve the performance of the ARIMA model on collision count data in Great Britain. Recently, [16] reported that most studies use monthly or yearly data, and only few studies analyze the impact of weather conditions on daily car crash counts. Using metereological data from the

*Address correspondence to this author at the Department of Mechanical and Industrial Engineering, University of Toronto, 5 King's College Road, Toronto, ON, M5S 3G8, Canada; Tel:+1 416 9784184;

Fax:+1 416 9787753; E-mail: Makis@mie.utoronto.ca
Netherlands, the authors analyzed the daily crash data using a time-series approach. Although the time series approach allows for time-dependent stochastic explanatory variables, researchers have found the approach to be overly complicated and even problematic when predicting future accidents. For example, recent studies of [17] and [9] concluded that time-varying covariates are difficult to account for, and subsequent findings are even more difficult to interpret.

In this paper, we take a different perspective than the two aforementioned approaches. We propose a continuous-time stochastic model to describe the relation between highway accidents and winter weather dynamics, which we believe to be a more natural way to describe discrete-event highway accidents that occur in continuous time. In particular, the accident counting process is modeled as a non-homogeneous Poisson process (NHPP) with an intensity function that depends on a (Markovian) weather process. Such a model is known in the stochastic process literature as a Markovmodulated Poisson process (MMPP) and has been successfully applied to queuing and telecommunications problems [18-23]. Generally, MMPP models are suitable when the systems and components function in a randomly changing environment. Thus, the MMPP is able to capture accidents with randomly changing weather conditions. The advantage this model has over standard regression models is its ability to handle autocorrelated, time-dependent stochastic covariates in continuous time. Furthermore, unlike the time series approach, it is able to provide explicit, closedform prediction formulae for both weather and accidents over any future time horizon (i.e. short or long-term 


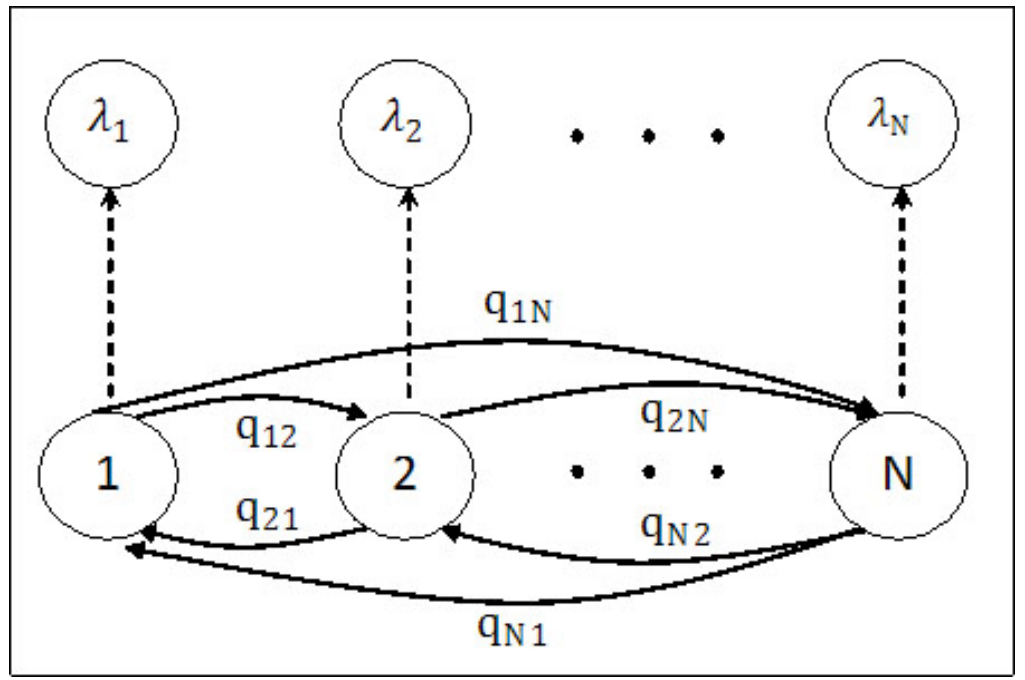

Fig. (1). Graphical illustration of the Markov-modulated Poisson process applied to highway accidents and weather dynamics. The bottom row is the (Markov) state diagram for the weather process. The top row shows the different possible crash intensities that depend on the current state of the weather.

predictions). This is an attractive feature in practice since predictions are extremely easy to implement and interpret. To our knowledge, MMPPs have never been applied to accident prediction problems under stochastically changing weather conditions.

We now provide an overview of the contributions and findings of this paper. In Section 2, we describe the stochastic MMPP model in detail. The likelihood function is expressed in a closed-form and maximum likelihood estimates are obtained for the model parameters. Predicting future accidents is then discussed through the so-called Operational Performance Function (OPF). Explicit closedform prediction formulae are developed for accidents over any future time horizon of length $h>0$ (i.e. short or longterm predictions). In Section 3, we describe the accident and winter weather data sets. In particular, we consider collision data during the winter months of 2000-2007 on Highway 401, the busiest highway in North America. The collision data comes from the Ministry of Transportation of Ontario (MTO) and the weather information data comes from Environment Canada. In Section 4, the MMPP model is applied to the real data sets, and MLE and prediction results are provided. Section 5 contains concluding remarks.

\section{STOCHASTIC MODEL AND METHODOLOGY}

In this section, we provide a detailed description of the Markov-modulated Poisson process (MMPP) and show how it can be used to model the accident and weather processes.

The MMPP assumes that collisions on a well defined highway segment follow a point process $\left(N_{t}: t \in \mathbb{R}_{+}\right)$with intensity function $t \mapsto \lambda\left(X_{t}\right)$ that depends on a weather process $\left(X_{t}: t \in R_{+}\right)$. The random variable $N_{t}$ represents the number of accidents that occur by time $t \in \mathbb{R}_{+}$. The random variable $X_{t}$ represents a categorical weather condition (e.g. snow, rain, haze, etc.) at time $t \in \mathbb{R}_{+}$that is common to all drivers and takes values in a discrete set $x=\{1, \ldots, N\}$. For each $x \in \mathcal{X}$, we write $\lambda_{x}:=\lambda(x)$.

The weather process $\left(X_{t}: t \in \mathbb{R}_{+}\right)$is assumed to follow a continuous-time homogeneous Markov chain with generator $\mathbb{Q}=\left(q_{x y}\right)_{N \times N}$ and initial distribution $\alpha_{x}=P\left(X_{0}=x\right)$, $x \in \mathcal{X}$. The dynamics of the the MMPP are graphically illustrated in Fig. (1).

Let $\Lambda=\left(\lambda_{1}, \ldots, \lambda_{N}\right)^{\prime}$ be the vector of possible intensities and $\Theta=\left(\alpha_{1}, \ldots, \alpha_{N}\right)^{\prime}$ be the initial weather distribution. Then, the (unknown) parameters of our model that need to be estimated are:

$\Lambda=\left(\lambda_{1}, \lambda_{2}, \ldots, \lambda_{N}\right)$,

$Q=\left(\begin{array}{cccc}q_{11} & q_{12} & \ldots & q_{1 N} \\ q_{21} & q_{22} & \ldots & q_{2 N} \\ \vdots & \vdots & \vdots & \vdots \\ q_{N 1} & q_{N 2} & \ldots & q_{N N}\end{array}\right)$,

$\Theta=\left(\alpha_{1}, \alpha_{2}, \ldots, \alpha_{N}\right)$

Our first objective is to determine the maximum likelihood estimates (MLEs) for unknown parameters in sets $\Lambda, \mathbb{Q}$ and $\Theta$.

\subsection{Maximum Likelihood Estimation}

Both the accident process $\left(N_{t}\right)$ and weather process $\left(X_{t}\right)$ are continuously observed over a fixed period $\left[0, s_{n}\right]$, where $s_{n}$ is the time of the $n$th transition of the weather process $\left(X_{t}\right)$. During the interval $\left[0, s_{n}\right]$, we denote the weather observation sample as 
$x=\left\{\left(x_{0}, t_{0}\right), \ldots,\left(x_{n-1}, t_{n-1}\right), x_{n}\right\}$, where $x_{m}$ is the $m$ th state the weather process passes through, and $t_{m}$ is the sojourn time the weather process remains in state $x_{m}$, i.e. $t_{0}+\cdots+t_{n-1}=s_{n}$. The accident observations are denoted $u=\left(u_{1}, \ldots, u_{L}\right)$, where $0 \leq u_{1}<\cdots<u_{L} \leq s_{n}$ are the time points of $L$ observed accidents during the interval $\left[0, s_{n}\right]$.

We now have the likelihood function (see e.g. [21]):

$$
\begin{gathered}
L(\Lambda, \mathbb{Q}, \Theta \mid u, x)=\prod_{k=1}^{L} \lambda_{\omega\left(u_{k}\right)} \cdot \exp \left(-\sum_{m=0}^{n-1} t_{m} \lambda_{x_{m}}\right) \\
\cdot \alpha_{x_{0}} \prod_{x \neq y} q_{x y}^{n} \prod_{w \in x} e^{q_{w w} w^{\tau} w}
\end{gathered}
$$

where $\omega\left(u_{k}\right) \in x$ is the observed state of the weather process at time $u_{k}, n_{x y}$ is the transition frequency of $x \rightarrow y$ in the sample $x_{0}, x_{1}, \ldots, x_{n}$, and $\tau_{w}$ is the total observed time for which the weather process occupied state $w$ during the interval $\left[0, s_{n}\right]$.

The log likelihood function is obtained as follows.

$$
\begin{aligned}
\log L(\Lambda,, \Theta \mid u, x) & =\sum_{k=1}^{L} \log \lambda_{\omega\left(u_{k}\right)}-\sum_{m=0}^{n-1} t_{m} \lambda_{x_{m}} \\
& +\log \alpha_{x_{0}}+\sum_{x \neq y} \log q_{x y}^{n_{x y}}+\sum_{w \in x} q_{w w} \tau_{w}
\end{aligned}
$$

The MLEs can then be found by maximizing equation (5) with respect to each parameter in sets $\Lambda, \mathbb{Q}$ and $\Theta$. Using standard univariate optimization arguements it is not difficult to show that the MLEs are given as follows:

$$
\begin{aligned}
& \hat{\lambda}_{x}=\frac{n_{x}(u)}{\tau_{x}}, \quad \forall x \in x, \\
& \hat{q}_{x y}=\frac{n_{x y}}{\tau_{x}}, \quad \forall x \neq y \in x, \\
& \hat{q}_{w w}=-\sum_{z \neq w} q_{w z}^{*}, \quad \forall w \in x, \\
& \hat{\alpha}_{x_{0}}=1, \\
& \hat{\alpha}_{x}=0, \quad \forall x \in x \backslash\left\{x_{0}\right\},
\end{aligned}
$$

where $n_{x}(u)=\left|\left\{k: \omega\left(u_{k}\right)=x\right\}\right|$ is the number of times $\omega\left(u_{k}\right)=x$ in the first sum on the right hand side of (5).

\subsection{Accident Predictions And The Operational Performance Function}

Once the MLEs have been obtained using equation (6), we can move to predicting the expected frequency of accidents over any future time horizon $h>0$. Since the accident process $\left(N_{t}\right)$ and the weather process $\left(X_{t}\right)$ are observed over a fixed time interval $[0, s]$, we are interested in the evaluating the following conditional expectation:

$O_{s}(h):=E\left(N_{s+h}-N_{s} \mid N_{u}, X_{u} ; 0 \leq u \leq s\right)$,

which represents the expected number of accidents that will occur over the next $h$ time units, given all accident and weather information until time $s$. We can think of $O_{s}(h)$ as a function of $h$, i.e. $h \mapsto O_{s}(h)$. This function is known as the operational performance function (OPF).

In terms of prediction, the goal is to give an explicit closed-form expression for the OPF defined in (7). We have the following result. For any $s, h>0$, the OPF can be explicitly computed via the following equation:

$O_{s}(h)=\sum_{y \in x} \hat{\lambda}_{y} \int_{0}^{h} \hat{p}_{x_{y}}(u) d u$,

where $\hat{p}_{x y}(u)=P\left(X_{u}=y \mid X_{0}=x\right), x, y \in X$, are computed using the MLEs $\hat{\mathbb{Q}}$ and $\hat{\Theta}$ given in equation (6). Equation (8) shows that accident predictions can be computed explicitly over any future time horizon (i.e. for any value of $h$ ). Therefore, our model works well for both short and longterm accident predictions. In the next section, we describe the winter weather data set provided by the Ministry of Transportation of Ontario (MTO).

\section{DATA DESCRIPTION}

The site specific database provided by Ministry of Transportation of Ontario (MTO) includes the motor vehicle collision database (MVAB) from 2000-2007 in Ontario. The weather data set comes from Environment Canada. The MVAB data from 2000-2007 was carefully examined and the highway segment with the highest winter collision frequency was selected for this study. Based on the analysis, Highway 401, starting from Highway 404 and ending at James Snow Parkway (segment number: 47635 - 47695) was selected for the MMPP analysis (see Fig. (2).

From Environment Canada, eight main types of weather conditions were reported during the winter months of 2000-

Table 1. Winter Weather Conditions Reported by Environment Canada

\begin{tabular}{|c|c|}
\hline Weather Index $(\boldsymbol{x})$ & Description of Weather Condition \\
\hline \hline$x=1$ & clear \\
\hline$x=2$ & cloudy \\
\hline$x=3$ & rain \\
\hline$x=4$ & snow \\
\hline$x=5$ & drizzle \\
\hline$x=6$ & ice \\
\hline$x=7$ & fog \\
\hline$x=8$ & haze \\
\hline
\end{tabular}




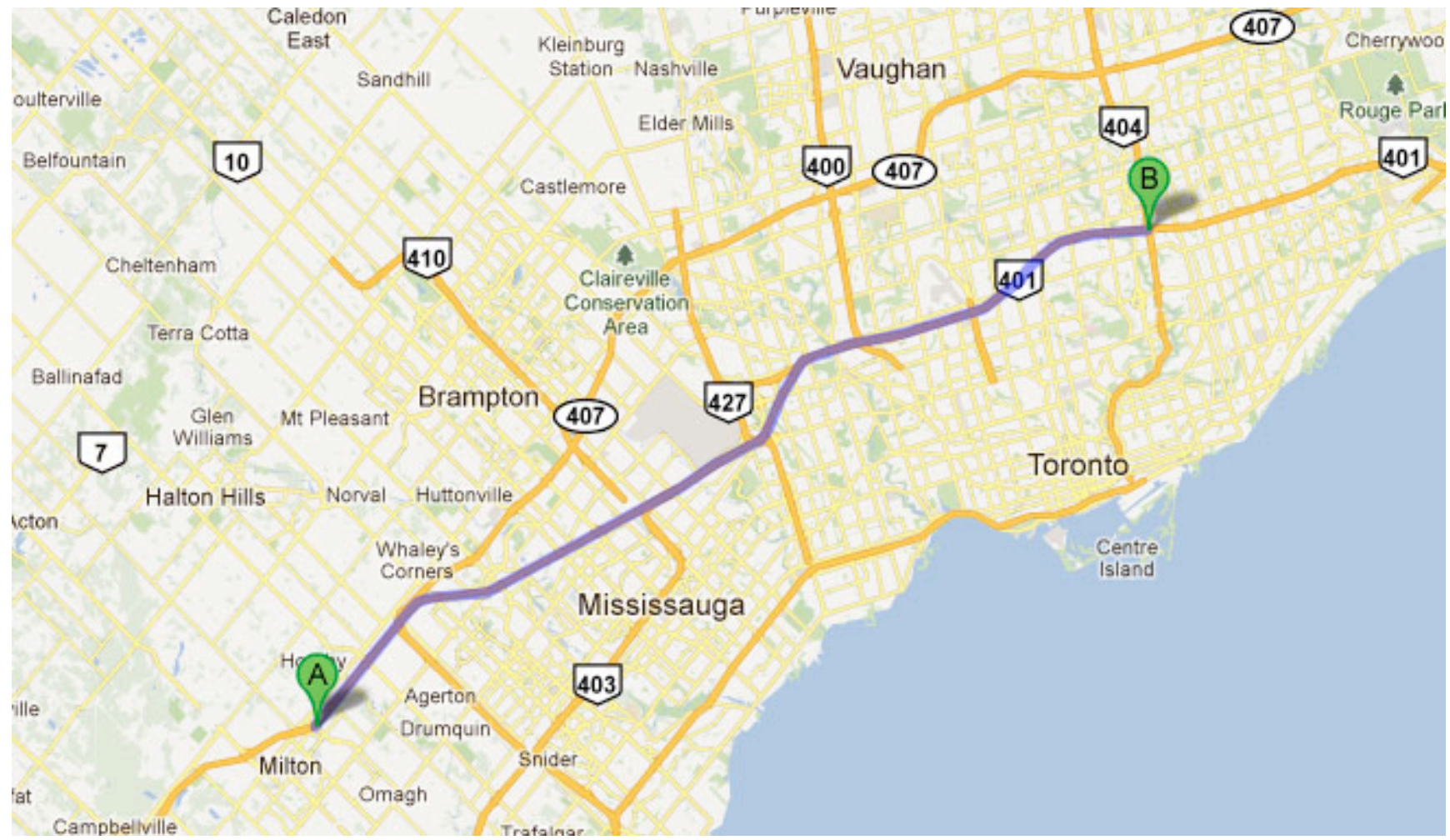

Fig. (2). Highway 401, starting from Highway 404 and ending at James Snow Parkway, had the highest winter accident frequency and was selected for the MMPP analysis.

2007. The eight identified weather contions (and their respective indices) are summarized in Table 1. In the next section, the MMPP model is applied to the real highway accident and winter weather data. Data from years 20002006 are used as training data and the winter data of 2007 is used to evaluate our model predictions.

\section{RESULTS}

In this section, we apply the MMPP results developed in Section 2 on the data set described in Section 3. In subsection 4.1, we first discuss reducing the number of weather states $N$ (and hence the number of model parameters) using a novel clustering approach. In subsection 4.2, MLEs are obtained using winter data from 2000-2006 and goodness-of-fit tests are performed. In subsection 4.3, we use the fitted model based on the 2000-2006 winter data to make predictions for winter data from 2007 using the Operational Performance Functions (OPFs) defined in equation (8).

\subsection{Determining the Number of Weather States $N$}

In this subsection, we discuss how to determine the appropriate number of weather states $N$. Although Environment Canada categorized weather states into eight main conditions, to avoid over parameterization, it is possible to "cluster" conditions with similar statistical properties to define a weather process with $N<8$ states. The simplest division of the weather states is to cluster precipitation and non-precipitation conditions as a MMPP(2) model Ramesh. Recall, from the MLEs given in equations (6), the estimates for the intensity rates $\lambda_{x}$ are given by the statistic $\frac{n_{x}(u)}{\tau_{x}}$. We plot this statistic for each possible weather condition and obtain the plot in Fig. (3).

Fig. (4) shows that there are natural "clusters" of weather states that have different intensities of collisions. We apply the K-mean clustering algorithm and determine that there are three natural groups of weather states with significantly different collision intensities.

After classifying the weather conditions, we define the weather process $\left(X_{t}: t \in \mathbb{R}_{+}\right)$to have state space $X=\{1,2,3\}$ with state interpretations given in Table 2 .

\subsection{Maximum Likelihood Estimates and Goodness-of-Fit Test}

In this subsection, we determine the maximum likelihood estimates of the model parameters. The weather process $\left(X_{t}: t \in \mathbb{R}_{+}\right)$is assumed to have state space $X=\{1,2,3\}$ defined in Table 2 . Using equation (6), the maximum

Table 2. Clustered Weather Conditions Based on the K-means Clustering Algorithm

\begin{tabular}{|c|c|}
\hline Weather Index $(\boldsymbol{x})$ & Description of Weather Condition \\
\hline \hline state 1 & clear, cloudy, rain, fog and haze \\
\hline state 2 & snow, drizzle \\
\hline state 3 & ice \\
\hline
\end{tabular}


Table 3. $95 \%$ confidence Intervals for the Intensity Estimates

\begin{tabular}{|c|c|c|c|}
\hline & $\lambda_{1}$ & $\lambda_{2}$ & $\lambda_{3}$ \\
\hline \hline MLEs & 0.6803 & 1.334 & 2.7 \\
\hline $95 \% \mathrm{CI}$ & \pm 0.0024 & \pm 0.012 & \pm 0.61 \\
\hline
\end{tabular}

Table 4. $p$-Value of the Laplace Test, which Confirm Constant Intensity Rates

\begin{tabular}{|c|c|c|c|}
\hline & $\lambda_{1}$ & $\lambda_{2}$ & $\lambda_{3}$ \\
\hline \hline$U_{x}$ & -1.2500 & 0.76 & 0.3192 \\
\hline$p$-value & 0.2113 & 0.4473 & 0.7496 \\
\hline
\end{tabular}

Table 5. Collision Frequency Comparison

\begin{tabular}{|c|c|c|c|c|c|}
\hline & & 3 Hours & 6 Hours & 12 Hours & 24 Hours \\
\hline \hline \multirow{2}{*}{ State 1 } & predicted & 2.15 & 4.42 & 9.07 & 7.5923 \\
& actual & 1.87 & 3.7531 & 11.12 & 15.673 \\
\multirow{2}{*}{ State 2 } & predicted & 3.43 & 6.2 & 14.713 & 26.159 \\
& actual & 3.7478 & 7.5942 & 13.2 & 21.247 \\
\hline \multirow{2}{*}{ State 3 } & predicted & 5.274 & 8.28 & 21.846 & 22.61 \\
& actual & 7.4615 & 13 & 29.692 \\
\hline
\end{tabular}

likelihood estimates of parameters are given by:

$\hat{\Lambda}=(0.6803,1.334,2.70)$,

$\widehat{Q}=\left(\begin{array}{ccc}-0.0463 & 0.0444 & 0.0019 \\ 0.2699 & -0.2769 & 0.007 \\ 0.3509 & 0.2188 & -0.5697\end{array}\right)$

$\hat{\Theta}=(1,0,0)$.

We next validate the model assumption made by the MMPP that accident rates are constant (i.e. have no other dependencies) in a given weather state. We first compute the $95 \%$ confidence interval (CI) for the MLEs of the intensity rates. Table 3 shows that the CIs are very tight, which indicates that the constant intensity assumption is reasonable. To test this hypothesis more rigorously, we perform the Laplace test (see e.g. [21]) which tests the follow null hypothesis:

$H_{0}$ : the underlying point process is a homogeneous Poisson process

$H_{1}$ : the underlying point process is a non-homogeneous Poisson process with increasing/decreasing density.

The test statistic is given by

$$
U_{x}=\frac{\sum_{i=1}^{n_{t}} t_{i}-\frac{1}{2} n_{t} T}{T \sqrt{\frac{n_{t}}{12}}}, x=1,2,3
$$

which follows a t-distribution under null hypothesis. The corresponding $\mathrm{p}$-values for each of the three intensities are given in Table 4. The p-values support hypothesis that the collision rate is constant in each of the weather states.

\subsection{Performance Testing via the OPF}

In this subsection, we use the 2007 year winter data (December, 01st, 2006 to March, 31th, 2007) to test the MMPP model prediction capabilities. The predicted value (i.e. OPF) is calculated using equation (8), and represents the expected number of collisions over a future interval of length $h$ given the current weather condition. We consider different time intervals of length $\mathrm{h}=3,6,12,24$ hours.

The predicted values are compared with actual 2007 winter collision data. The way we compute the actual average number of collisions over a future interval of length $\mathrm{h}=3,6,12,24$ hours is as follows. We first divide the entire winter season of 2007 into segments of length $h$. At the beginning of each segment we identify the current weather state $\mathrm{x}=1,2,3$. We then compute the total number of collisions that occur during segments that start in weather condition $x$ and jump to weather condition $j$ (where $\mathrm{j} \neq \mathrm{i}$ ), and divide by the total number of such segments.

Table 5 and Fig. (5) show forecasting results for different forecasting lengths (i.e. $\mathrm{h}=3,6,12,24$ hours) starting in different weather conditions, $x=1,2,3$. The results show that the model is able to predict future collision frequencies well, and predictions are particularly good for the short time lengths $h$.

We next compare our 3-hour prediction results with a standard homogeneous Poisson model, which assumes that the collision counting process follows a homogeneous Poisson process with parameter $\lambda$ that does not change with the weather process. The result is shown in Fig. (6) with predicted value as 2.4453 . 


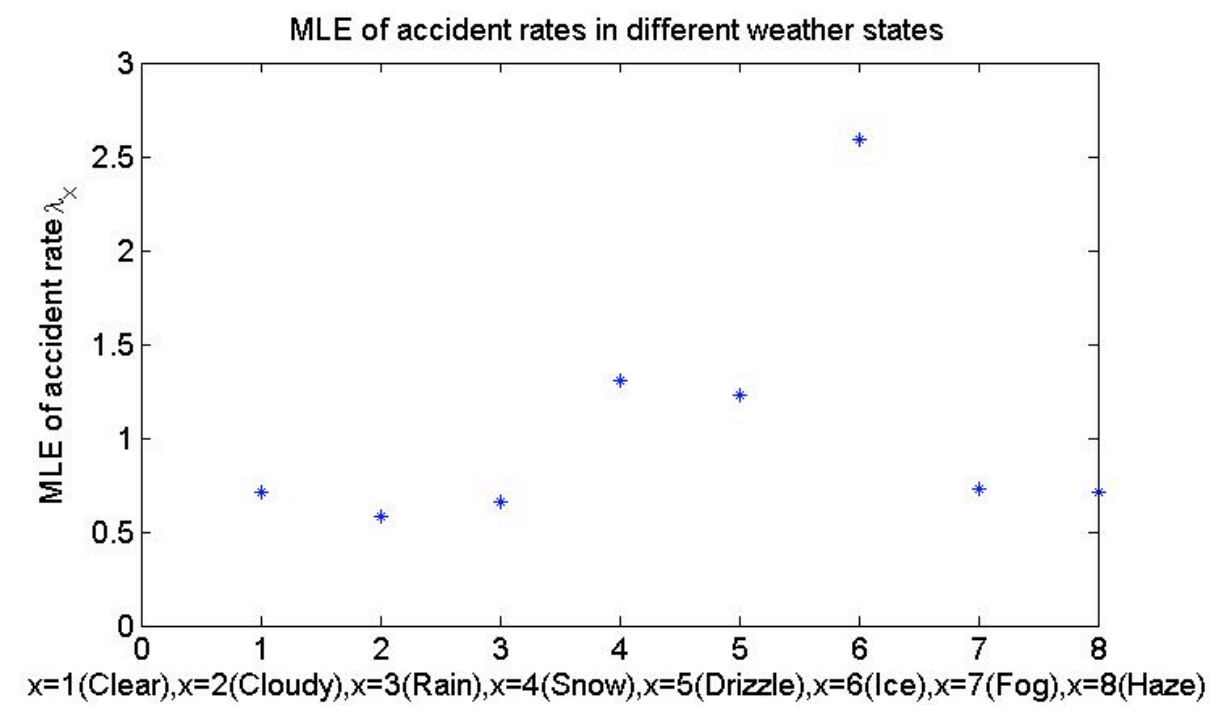

Fig. (3). Accident rate in each weather conditions.

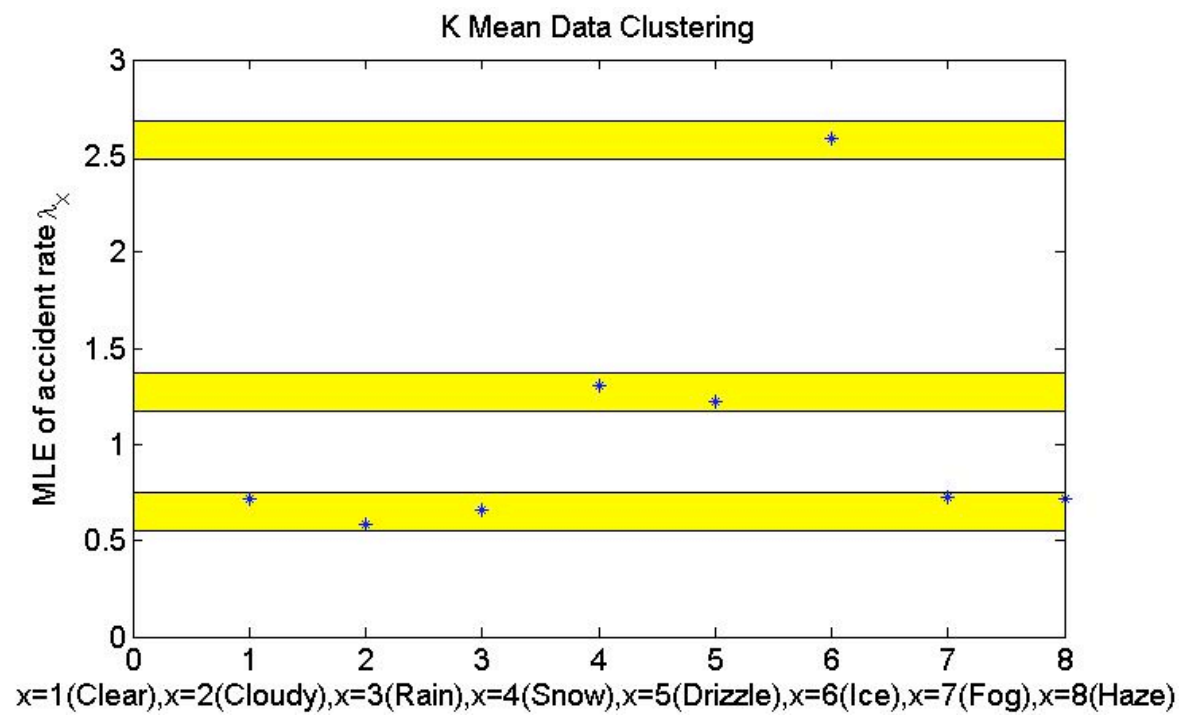

Fig. (4). MLEs shows three distinct weather status using the K-means clustering algorithm.

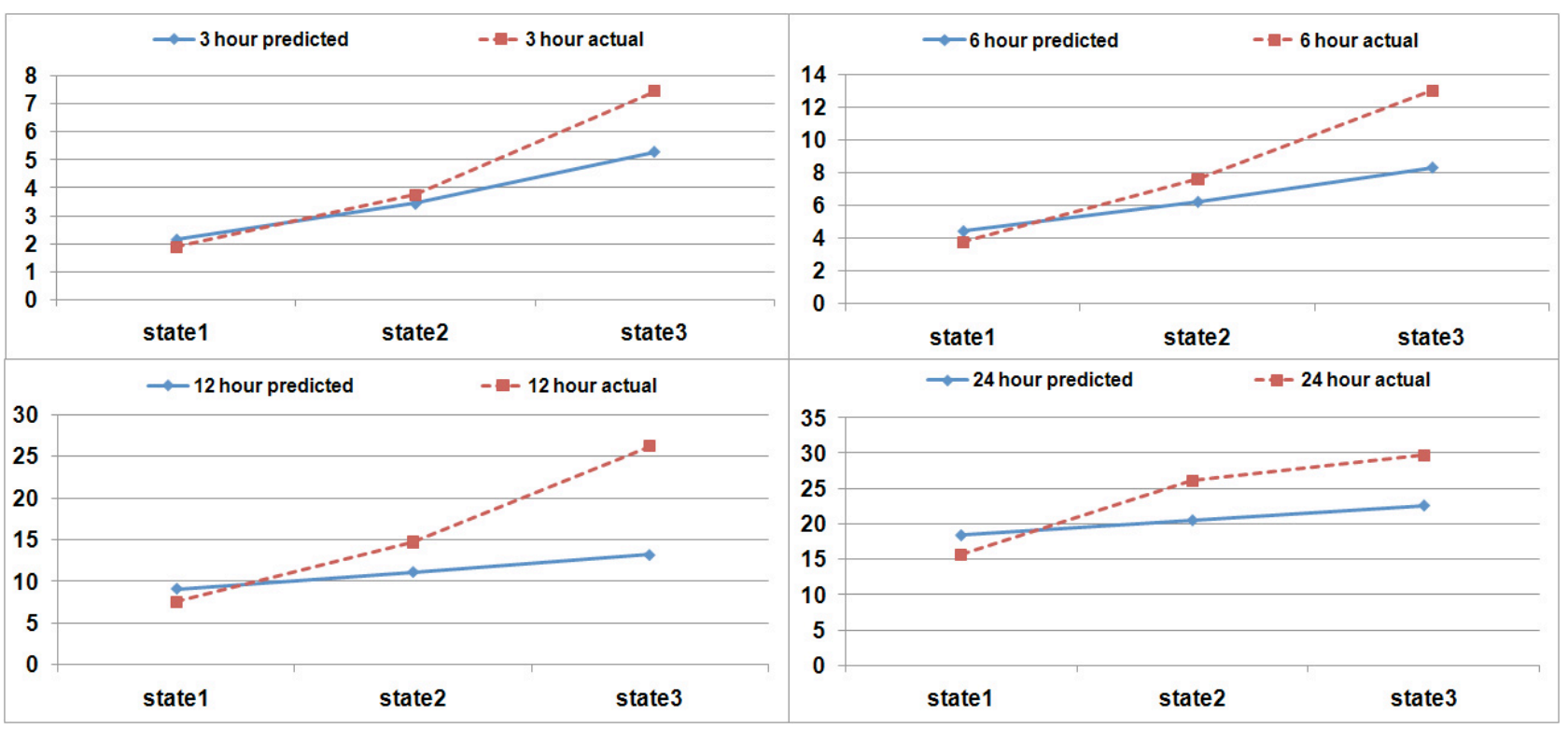

Fig. (5). Three hours prediction comparison of dynamic MMPP and Poisson model. 


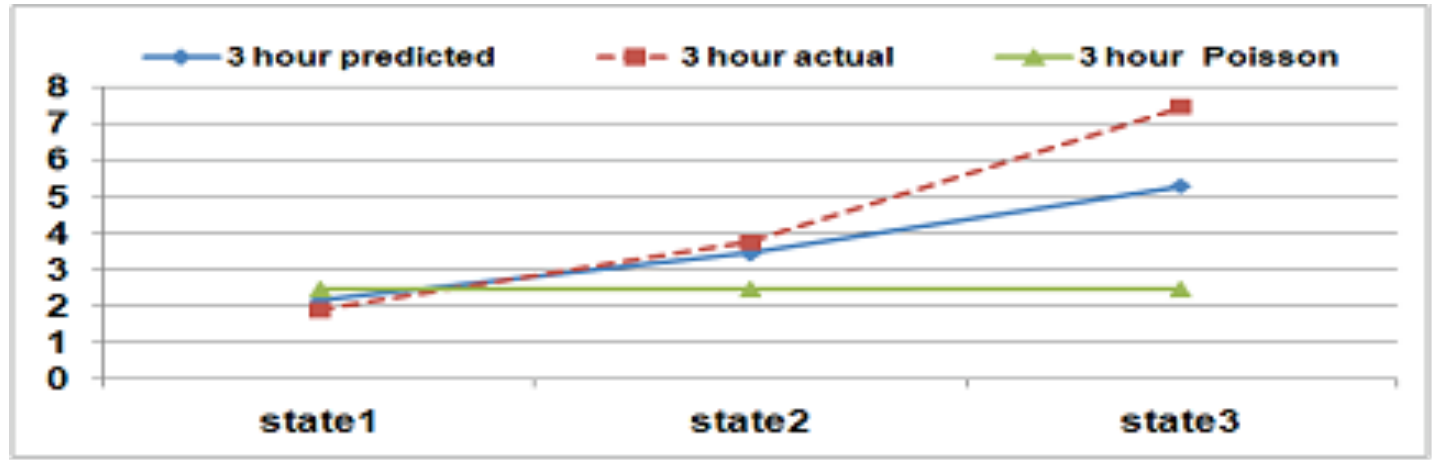

Fig. (6). Three hours prediction comparison of dynamic MMPP and Poisson model.

Fig. (6) clearly illustrates the benefits of our dynamic MMPP. Since the standard Poisson model is not a dynamic model, when the weather process enters states two and three, the collision frequency is severely underestimated. This suggests that using a weather-dependent dynamic model will provide much better predictions for collision frequencies.

\section{CONCLUSIONS}

In this paper, we considered the problem of modeling and predicting highway accidents in the presence of randomly changing winter driving conditions. Unlike most ccident prediction models in the literature, we proposed a continuous-time stochastic model to describe the relation between highway accidents and winter weather dynamics.In particular, the accident and weather processes were modeled as a Markov-modulated Poisson process (MMPP). One main advantage of our approach is that it provides explicitly closed-form prediction formulae for both weather and accidents over any future time horizon (i.e. short or longterm predictions). It was shown that the likelihood function, maximum likelihood estimates, and operational performance functions all have closed-form expressions. To illustrate the effectiveness of the proposed stochastic model, we studied a large winter data set provided by Ministry of Transportation of Ontario (MTO) that includes motor vehicle accidents on Highway 401. Our results indicate that the MMPP model performs well for predicting accidents, and provides much better predictions for collision frequencies compared with the standard and static Poisson model.

\section{CONFLICT OF INTEREST}

The authors confirm that this article content has no conflicts of interest.

\section{ACKNOWLEDGEMENTS}

Declared none.

\section{REFERENCES}

J.M. Ma, K.M. Kockelman, and P. Damien, "A multivariate Poisson-lognormal regression model for prediction of crash counts by severity using Bayesian methods". Accident Analysis and Prevention, vol. 40, no. 3, pp.964-975, 2008.

[2] N.V. Malyshkina and F.L. Mannering, "Zero-state Markov switching count-data models: an empirical assessment". Accident Analysis and Prevention, vol. 42, no.1, pp. 122-130, 2010.

[3] B.J. Park, D. Lord, and J.D. Hart, "Bias properties of Bayesian statistics in finite mixture of negative regression models for crash data analysis". Accident Analysis and Prevention, vol. 42, no. 2, pp. 741-749, 2010.

[4] J. Hakjin, L. Soobeom, and W.K. Seong, "Bayesian analysis for zero-inflated regression models with the power prior: Applications to road safety countermeasures". Accident Analysis and Prevention, vol. 42, no. 2, pp. 540-547, 2010.

[5] P.Ch. Anastasopoulos and F.L. Mannering, "A note on modeling vehicle accident frequencies with random-parameters count models". Accident Analysis and Prevention, vol. 41, no.1, pp.153$159,2009$.

[6] P. Sunil, G. Srinivas Reddy, and D. Lord, "Analysis of crash severities using nested logit model - Accounting for the underreporting of crashes". Accident Analysis and Prevention, vol. 45, pp. 646-653, 2012.

[7] S.R. Geedipally, D. Lord, and S.S. Dhavala, "The negative binomial-Lindley generalized linear model: Characteristics and application using crash data". Accident Analysis and Prevention, vol. 45, pp. 258-265, 2012.

[8] D. Lord, S.P. Washington, and J.N. Ivanc, "Poisson, Poissongamma and Zero-inflated Regression Models of Motor Vehicle Crashes: Balancing Statistical Fit and Theory". Accident Analysis and Prevention, vol. 37, pp. 35-46, 2005.

[9] D. Lord and F. Mannering, "The Statistical Analysis of CrashFrequency Data: A Review and Assessment of Methodological Alternatives". Transportation Research Part A: Policy and Practice, vol. 44, no. 5, pp. 291-305, 2010.

[10] M.A. Quddus, "Time series count data models: An empirical application to traffic accidents". Accident Analysis and Prevention, vol. 40 , no. 5 , pp.1732-1741, 2008.

[11] Y.J. Kweon, S.E. Hartman, and C.W. Lynn, "Stop versus yield on pedestrian-involved fatal crashes in the United States". Accident Analysis and Prevention, vol. 41 no. 5, pp. 1034-1039, 2009.

[12] M. Ramsted, "Alcohol and fatal accidents in the United States: A time series analysis for 1950 - 2002". Accident Analysis and Prevention, vol. 40, no. 4, pp.1273-1281, 2008.

[13] A.I. McLeod and E.R. Vingilis, "Power computations in time series analyses for traffic safety interventions". Accident Analysis and Prevention, vol. 40 no. 3, pp. 1244-1248, 2008.

[14] X.P. Zheng and M. Liu, "An overview of accident forecasting methodologies". Journal of Loss Prevention in the Process Industries, vol. 22 no. 4, pp. 484-491, 2009.

[15] F.V.D. Bossche, G. Wets, and T. Brijs, "A regression model with ARIMA errors to investigate the frequency and severity of road traffic accidents". 83rd annual Meeting of the Transportation research Board. Washington, DC, USA, 2004.

[16] T. Brijs, D. Karlis, and G. Wets, "Studying the effect of weather conditions on daily crash counts using a discrete time-series model". Accident Analysis and Prevention, vol. 40, no. 3, pp. 11801190, 2008.

[17] S.P. Washington, M.G. Karlaftis, and F.L. Mannering, Statistical and Econometric Methods for Transportation Data Analysis, $2^{\text {nd }}$ ed. Chapman Hall/CRC: Boca Raton, FL, 2010.

[18] W. Fischer and M.H. Kathleen, "The Markov-modulated Poisson process (MMPP) cookbook". Performance Evaluation, vol. 18, no. 2, pp. 149-171, 1993.

[19] M.F. Neuts, Structured Stochastic Matrices of M/G/1 Type and Their Applications, CRC Press: New York, 1989. 
[20] S. Ozekici, and R. Soyer, "Semi-Markov modulated Poisson process: probabilistic and statistical analysis". Mathematical Methods of Operations Research, vol.64 no.1, pp. 125-144, 2006.

[21] I.V. Basawa, and P. Rao, Statistical Inference for Stochastic Processes. Academic Press, New York, 1980.
[22] N.I. Ramesh, "Statistical analysis on Markov Modulated Poisson process". Environmetrics, vol. 6, pp. 165-179, 1995.

[23] B. Lan and B. Persaud, "Evaluation of multivariate Poisson log normal fully Bayesian methods", Proceedings of the Paper Presented at the 89th Annual Meeting of the Transportation Research Board. USA, 2010.

Received: May 25, 2013

Revised: June 15,2013

Accepted: July 25, 2013

(C) Lin et al.; Licensee Bentham Open.

This is an open access article licensed under the terms of the Creative Commons Attribution Non-Commercial License (http://creativecommons.org/licenses/by$\mathrm{nc} / 3.0 /$ ) which permits unrestricted, non-commercial use, distribution and reproduction in any medium, provided the work is properly cited. 